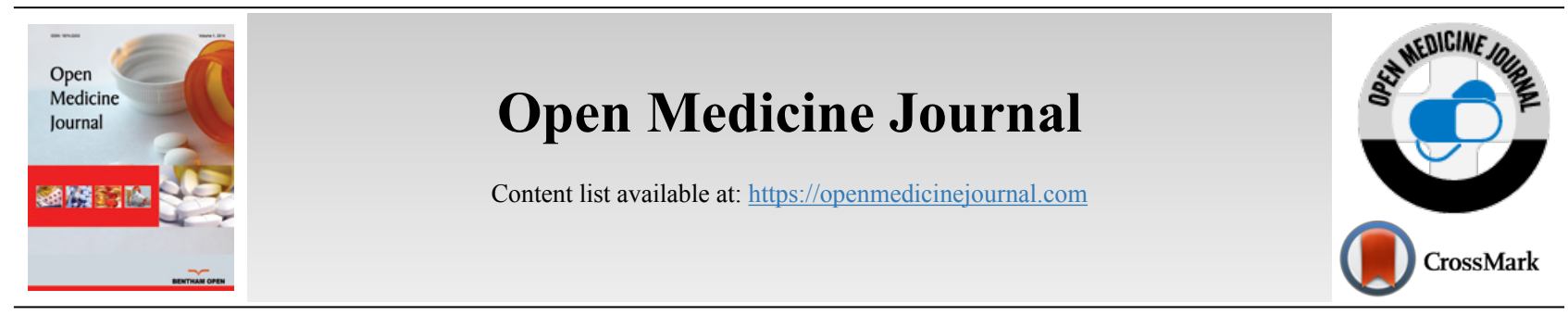

RESEARCH ARTICLE

\title{
Interaction between Urinary 11 Dehydrothromboxane B2 and Some Other Risk Factors in the Occurrence of Cerebral Infarction
}

\author{
Wang Lingling ${ }^{1}$, Chen Guixin ${ }^{1}$, Li Wei ${ }^{1}$ and Sun Hua ${ }^{2, *}$ \\ ${ }^{1}$ Department of Neurology, Affiliated Hospital of Beihua University, Jilin, 132011, China \\ ${ }^{2}$ Department of Endocrinology, Affiliated Hospital of Beihua University, Jilin, Jilin, 132001, China
}

\begin{abstract}
:
Objective:

The objective of this study is to analyze the interaction between the urinary 11-dehydrothromboxane B2 (11-DH-TXB2) content and the smoking, drinking, hypertension or diabetes history of patients with cerebral infarction, and to determine the value of 11-DH-TXB2 in the occurrence and prevention of cerebral infarction.

Methods:

117 patients with cerebral infarction and 50 healthy controls were selected. Their general information, including smoking, drinking, hypertension and diabetes history, was analyzed, and their urinary11-DH-TXB2 contents were measured. The difference in the urinary 11-DH-TXB2 content between the two groups, and the additive model of the interaction between the urinary 11-DH-TXB2 content and other risk factors of cerebrovascular diseases were statistically analyzed.

Results:

There was no significant difference in the general information between the two groups $(P>0.05)$. Significant differences were observed in the urinary 11-DH-TXB2 content, and the smoking, diabetes and hypertension history between the two groups, while there was no significant difference in the drinking history between the two groups. The analysis of the interaction additive model showed that the OR value was 1 , the synergy index (S) of increased 11-DH-TXB2 and smoking history was 1.219, the $\mathrm{S}$ of increased 11-DHTXB2 and hypertension history was 5.578 and that of increased 11-DH-TXB2 and the diabetes history was 4.604.

Conclusion:

There is a correlation between the urinary 11-DH-TXB2 content and the occurrence of cerebral infarction, and there is a synergistic interaction between the urinary DH-TXB2 content and the smoking, hypertension or diabetes history of patients with cerebral infarction. The increased 11DH-TXB2 may be a risk factor for cerebral infarction and the urinary 11-DHTXB2 content can be used as an index to predict the occurrence and progression of cerebral infarction.
\end{abstract}

Keywords: Urine 11-dehydrothromboxane B2, Thromboxane A2, Cerebral infarction, Risk factors, Hypertension, Synergy index.

\begin{tabular}{l|l|l|l} 
Article History & Received: July 12, 2019 & Revised: October 10, 2019 & Accepted: October 16, 2019
\end{tabular}

\section{INTRODUCTION}

Cerebral infarction is a common disease with high incidence and irreversible damage to neurological function caused by cerebral ischemia and anoxic necrosis due to various reasons [1]. In cerebral infarction, thromboxane A2 (TXA2) can induce platelet aggregation, and vascular smooth muscle contraction and proliferation, by activating its specific TXA2

\footnotetext{
* Address correspondence to this author at the Department of Endocrinology, Affiliated Hospital of Beihua University, Jilin, Jilin, 132011, China; Tel: 15643217009; E-mail: 373049083@qq.com
}

receptors, playing a guiding role in the occurrence and development of cerebral infarction [1]. 11-DH-TXB2 (11dehydrothromboxane $\mathrm{B}_{2}$ ) is a metabolite of serum TXA2 and can indirectly reflect the content of TXA2 in the serum since TXA2 is an active substance of platelet formation and its level can reflect the activation level of platelets in vivo [1]. Therefore, whether the change of TXB2 content in the serum of patients is correlated with the occurrence of cerebral infarction and TXB2 can be regarded as one of the risk factors for the primary prevention of cerebral infarction, which 
remains to be discussed. In this study, the urinary 11-DHTXB2 content of patients with cerebral infarction, and the interaction between urinary 11-DH-TXB2 and some other risk factors of cerebral infarction were retrospectively analyzed to provide a scientific basis for the urinary 11-DH-TXB2 as a risk factor for the primary prevention of cerebral infarction.

\section{MATERIALS AND METHODS}

\subsection{Research Objects}

The patients admitted to the Department of Neurology and Physical Examination, the affiliated Hospital of Beihua University from November 2016 to October 2018 were selected. The subjects were divided into a cerebral infarction group and a non-cerebral infarction group by a non-randomized control study. The diagnosis of patients in the cerebral infarction group met the diagnostic criteria of cerebrovascular diseases established by the Neurology Branch of the Chinese Medical Association [1]. The patients were diagnosed by CT or MRI and the NIHSS (National Institute of Health Stroke Scale) scores were from 5 to 10 points. The midstream urine samples of all the patients included in the cerebral infarction group were collected within 24 hours of the attack of cerebral infarction for measuring the content of 11-DH-TXB2 in the urine. The exclusion criteria for patients in both the groups were those who had taken antiplatelet, anticoagulant and thrombolytic drugs that could affect the function of platelets within a month.

\subsection{Determination and Judgement Criteria of Urinary 11- DH-TXB2}

The urine samples of all the subjects in both the groups were the midstream urine samples collected the next morning and the content of 11-DH-TXB2 was determined by the enzyme-linked immunosorbent assay (ELISA). Criteria for judgement: urinary 11-DH-TXB2 content less than $2500 \mathrm{pg} / \mathrm{mg}$ was considered a normal activity of urinary 11-DH-TXB2, and the content more than or equal to $2500 \mathrm{pg} / \mathrm{mg}$ exhibited increased activity of urinary 11-DH-TXB2 $(+)$.

\subsection{Assignment Standards}

The physical indexes and test results of all the patients were determined: gender $($ male $=1$, female $=2)$; hypertension, diabetes or smoking history $($ no $=0$, yes $=1)$; urinary $11-\mathrm{DH}$ TXB2 (less than $2500 \mathrm{pg} / \mathrm{mg}=0$, more than $2500 \mathrm{pg} / \mathrm{mg}=1$ ). The quantitative data included the age and urinary $11-\mathrm{DH}-$ TXB2 content.

\subsection{Statistical Method}

The SPSS20.0 software was used for the statistical analysis. The measurement data subject to normal distribution were expressed as mean \pm standard deviation $\left(\bar{x}^{ \pm s}\right)$ and the difference between the groups was compared by an independent sample $t$-test. The enumeration data were expressed by rate or constituent ratio and the difference between the two groups was compared by $\chi 2$ test. Non-conditional logistic regression analysis was used for the multivariate analysis.
Interaction additive model was used for the study of two-factor interaction: $\mathrm{R} 11-\mathrm{R} 00=(\mathrm{R} 01-1)+(\mathrm{R} 10-1)$. OR value was determined by the two factors of interaction. The odds ratio was obtained by the case-control study $(\mathrm{OR}=\mathrm{ad} / \mathrm{bc})$; calculation of relative excess risk of interaction (Excess relative risk, RERI), attributable proportion of interaction (Interaction Attribution Ratio,API) and synergy index (interaction index, $\mathrm{S})$. Calculation formulas: RERI=R11-(R01+R11)+1; API= [R11-(R01+R10)+1]/R11; S=OR11-1/ [(OR01-1)+(OR10-1)]. $P<0.05$ was considered a statistically significant difference.

\section{RESULTS}

\subsection{Demographics, Individual History, Past Medical} History and Urinary 11-DH-TXB2 Content of the Subjects

There were 117 patients in the cerebral infarction group (52 males and 65 females), aged from 30 to 87 years (61.4 \pm 11.3 years), and 50 patients ( 25 males and 25 females) in the non-cerebral infarction group, aged from 44 to 86 years $(60.0 \pm$ 10.0 years). There was no significant difference in the demographics of the subjects and in the history of alcohol consumption between the cerebral infarction group and noncerebral infarction group $(P>0.05)$, and there were significant differences in the smoking, hypertension and diabetes history $(P<0.05)$. The urinary $11-\mathrm{DH}-\mathrm{TXB} 2$ content of patients in the cerebral infarction group was significantly higher than that in the non-cerebral infarction group $(P<0.05)$, showing a difference in the 11-DH-TXB2 content in urine between the two groups, which indicates that 11-DH-TXB2 may be an indicator of urine changes in patients with early cerebral infarction. The results are shown in Table $\mathbf{1}$.

Table 1. Analysis of the general information of subjects.

\begin{tabular}{|c|c|c|c|}
\hline Variable & $\begin{array}{c}\text { Non-cerebral } \\
\text { infarction }\end{array}$ & $\begin{array}{c}\text { Cerebral } \\
\text { infarction }\end{array}$ & $\boldsymbol{P}$ \\
\hline Number of cases (n) & 50 & 117 & - \\
\hline Gender (male) & 25 & 52 & 0.509 \\
\hline Age (y) & $60.0 \pm 10.0$ & $61.4 \pm 11.3$ & 0.468 \\
\hline Smoking history & 9 & 41 & $0.028^{*}$ \\
\hline Drinking history & 13 & 31 & 0.947 \\
\hline Hypertension & 12 & 47 & $0.045^{*}$ \\
\hline Diabetes & 7 & 34 & $0.038^{*}$ \\
\hline 11-DH-TXB2(pg/mg) & $2083.0 \pm 1394.9$ & $3436.1 \pm 2786.3$ & $0.001^{*}$ \\
${ }^{*}: P<0.05$, pairwise comparison. & & \\
\end{tabular}

\subsection{Logistic Regression Analysis of the Urinary 11-DH-} TXB2 Level and Other Adverse Factors of Cerebral Infarction

The univariate analysis showed that the adverse factors of cerebral infarction included smoking, diabetes and hypertension history, and the urinary 11-DH-TXB2 content. The unconditional logistic regression analysis showed that smoking, diabetes and hypertension history, and the urinary 11DH-TXB2 content were positively correlated with the occurrence of cerebral infarction (OR $>1$, OR95\% excluding 1). The above factors are considered the strong risk factors for cerebral infarction Table 2. 
Table 2. The logistic between Risk factors and cerebral infarction.

\begin{tabular}{|c|c|c|c|c|c|c|}
\hline Conventional & Regression coefficient( $\boldsymbol{\beta})$ & Standard error & Wald & P & OR & 95\%CI \\
\hline Smoking history(\%) & 2.218 & 0.724 & 8.654 & 0.003 & 8.400 & $2.033-34.709$ \\
\hline Diabetes(\%) & 1.755 & 0.676 & 6.732 & 0.009 & 5.782 & $1.536-21.766$ \\
\hline Hypertension(\%) & 1.494 & 0.560 & 7.114 & 0.008 & 4.454 & $1.486-13.348$ \\
\hline $11-\mathrm{DH}-\mathrm{TXB}(\mathrm{pg} / \mathrm{mg})$ & 1.506 & 0.534 & 7.953 & 0.005 & 4.509 & $1.583-12.845$ \\
\hline
\end{tabular}

$*: P<0.05$, pairwise comparison.

Table 3. Interaction between increased 11-DH-TXB2 and other risk factors.

\begin{tabular}{|c|c|c|c|c|c|c|c|}
\hline \multirow[t]{2}{*}{ Variable } & \multicolumn{2}{|c|}{ No. of cases $(n)$} & \multirow[t]{2}{*}{ OR } & \multirow[t]{2}{*}{$95 \% \mathrm{CI}$} & \multirow[t]{2}{*}{ RERI } & \multirow[t]{2}{*}{ API } & \multirow[t]{2}{*}{$\mathbf{S}$} \\
\hline & $\begin{array}{c}\text { Non-cerebral } \\
\text { infarction }\end{array}$ & Cerebral infarction & & & & & \\
\hline \multicolumn{8}{|c|}{ TXB2/Smoking } \\
\hline$-/-$ & 36 & 19 & 1.00 & ref & \multirow[t]{4}{*}{2.71} & \multirow[t]{4}{*}{$16.80 \%$} & \multirow[t]{4}{*}{1.219} \\
\hline$+/-$ & 5 & 31 & 11.75 & $6.716-20.549$ & & & \\
\hline$-/+$ & 5 & 7 & 2.65 & $1.384-5.084$ & & & \\
\hline$+/+$ & 4 & 34 & 16.11 & $8.840-29.341$ & & & \\
\hline \multicolumn{8}{|c|}{ TXB2/ Hypertension } \\
\hline$-/-$ & 28 & 23 & 1.00 & ref & \multirow[t]{4}{*}{20.19} & \multirow[t]{4}{*}{$78.87 \%$} & \multirow[t]{4}{*}{5.578} \\
\hline$+/-$ & 7 & 27 & 5.20 & $3.136-8.619$ & & & \\
\hline$-/+$ & 10 & 9 & 1.21 & $0.710-2.072$ & & & \\
\hline$+/+$ & 5 & 38 & 25.60 & $11.787-55.637$ & & & \\
\hline \multicolumn{8}{|c|}{ TXB2/ Diabetes } \\
\hline$-/-$ & 31 & 11 & 1.00 & ref & \multirow[t]{4}{*}{63.98} & \multirow[t]{4}{*}{$77.33 \%$} & \multirow[t]{4}{*}{4.604} \\
\hline$+/-$ & 8 & 39 & 15.51 & $8.141-23.184$ & & & \\
\hline$-/+$ & 6 & 8 & 4.24 & $1.973-7.155$ & & & \\
\hline$+/+$ & 5 & 26 & 82.73 & $24.938-215.288$ & & & \\
\hline
\end{tabular}

OR values: The age, gender, smoking history, NIHSS scores on admission, hypertension, diabetes history, complications during hospitalization and urinary11-DH-TXB2 contents were adjusted.

\subsection{Analysis of the Addictive Model and Interaction of Increased Urinary 11-DH-TXB2 and Other Risk Factors}

The negative interference factor (-/-) was taken as the control, and the OR value was set as 1 in the interaction analysis. The results showed that (1) the RERI of the interaction between the increased urinary TXB2 content and the smoking history was 2.71 , indicating that the risk of cerebral infarction caused by the interaction between the increased TXB2 and the smoking history was increased to 2.7, the attributable proportion of interaction (API) was $16.80 \%$ and the synergy index (S) was 1.219 , indicating that the occurrence of cerebral infarction in $16.80 \%$ of the included patients might be due to the coexistence of TXB2 and smoking history, and the risk of cerebral infarction caused by the coexistence of the two factors was 1.219 times as much as that caused by either of them alone and there was a positive synergy of the two factors; (2) the RERI of the interaction between the increased urinary TXB2 content and the hypertension history was $20.19 \%$, indicating that the risk of cerebral infarction caused by the the increased TXB2 and the hypertension history was increased to 20.19 , the API was $78.87 \%$ and the $\mathrm{S}$ was 5.578 , indicating that $78.87 \%$ of the cerebral infarction in the included patients was caused by the coexistence of increased TXB2 and hypertension history, and the occurrence of cerebral infarction caused by the coexistence of the two factors was 5.578 times as much as that of either of them alone and there was a positive synergy of the two factors; (3) the RERI of the interaction between the increased urinary TXB2 content and the diabetes history was 63.98 , indicating that the risk of cerebral infarction caused by the interaction between the increased TXB2 and the diabetes history was increased to $77.33 \%$, the API was 63.98 and the S was 4.604 , indicating that $77.33 \%$ of the cerebral infarction in the patients was caused by the coexistence of increased TXB2 and diabetes history, and the risk of cerebral infarction caused by the coexistence of the two factors was 4.604 times as much as that caused by either of them alone and there was a positive synergy of the two factors (Table 3 ).

\section{DISCUSSION}

Cerebral infarction is a neurological disease, and the most important cause of pathogenesis that leads to atherosclerotic thrombus. In the course of the formation of atherosclerosis, the activated platelets can rapidly synthesize and release some active substances to affect the thrombogenesis, therefore the role of platelets in atherosclerosis is partially determined by scholars [1]. The relevant research results show that platelets can directly act as an "inflammatory cell" to participate in the formation of atherosclerosis [2]: (1) In the early stage, platelets mainly interact with monocytes and vascular endothelial cells, the platelets and monocytes adhere to each other to activate the monocytes, and then the monocytes further adhere to the vascular endothelial cells, driving the monocytes to migrate 
and accumulate in the intima of blood vessels; (2) In the late stage, platelets aggregate on the erosive and ruptured plaques to cause secondary thrombosis, thus leading to a series of acute or chronic clinical events. Therefore, the platelet is confirmed to be the only functional cell involved in the process of both inflammation and thrombosis. The content of 11-DH-TXB2, a TXA2 metabolic product, in the urine of the subjects, was measured in this study. It is well known that TXA2, the active substance produced by platelets, can influence the development of cerebral infarction by binding to its specific receptors, TXA2R. During the binding process of TXA2 and TXA2R, the calcium ions stored in the compact particles of platelets migrate to the cytoplasm to trigger the release of platelets, the activity of adenylate cyclase is inhibited, and the level of cAMP in the platelets decreases, which increases the functional activity of platelet adhesion, aggregation and release. Therefore, TXA2 strongly induces platelet aggregation and smooth muscle contraction, thereby resulting in increased blood viscosity and microcirculation disorders, playing an important role in the development of vascular lesions and thrombosis. On one hand, TXA2 induces platelet adhesion and aggregation, and on the other hand, it promotes the constriction and proliferation of vascular smooth muscles, resulting in the pathological changes of the cerebral ischemia-reperfusion injury [3], promoting atherosclerosis and affecting the stability of the plaque [4]. In normal cases, the content of TXA2 in the serum is very low $[5,6]$, while the content of TXA2 in the serum of patients with cerebral infarction significantly increases. However, since the half-life $\left(t_{1 / 2}\right)$ of TXA2 is short (about 30 seconds), TXA2 is rapidly converted to inactive TXB2, while 11-DH-TXB2 is a metabolite with long half-life formed by the metabolism of TXB2 in the liver and excreted out of the body in urine. It is produced by the activated platelets in the body, which is not easily influenced by the activation of platelets in vitro, therefore, the content of urinary 11-DH-TXB2 can better reflect the level of TXA2 in the body [7 - 12]. The results showed that the content of 11-DH-TXB2 in the urine of the patients with cerebral infarction was significantly higher than that of healthy people in the healthy control group, which was consistent with the results reported by Borg C [5]. Studies have shown that after cerebral infarction, ischemia and hypoxia of local tissues, and the damage and necrosis of cells due to the interruption of cerebral blood supply may result in the decomposition of a large amount of membrane phospholipids in the cell membrane, and the generation of TXA2 also increases, which aggravate cerebral ischemia and hypoxia. At the same time, the increase of serum TXA2 results in the strong contraction of vascular smooth muscles, thereby decreasing the cerebral blood flow, which further aggravates the ischemic brain injury [13].Studies have shown that atherosclerosis is closely related to the changes in the activity of TXB2 [14 - 16], and 11-DH-TXB2 is an independent risk factor for the carotid intima thickening in patients with diabetes and can be used as an indicator to predict vascular lesions in diabetic patients [17]. Relevant studies on the occurrence of cerebral infarction have found that more than half of the stroke incidence may be due to the risk factors, such as hypertension, diabetes, smoking, etc., and that the blood is in a hypercoagulable state and a partial inflammation at the same time in the course of cerebral infarction [18, 19]. The interaction between 11-DH-TXB2 and the smoking, hypertension or diabetes history was analyzed in this study. The results showed that (1) the RERI of the interaction between the increased urinary TXB2 content and the smoking history was 2.71 , indicating that the risk of cerebral infarction caused by the interaction between the increased TXB2 and the smoking history was increased to 2.7 , the attributable proportion of interaction (API) was $16.80 \%$ and the synergy index (S) was 1.219 , indicating that the occurrence of cerebral infarction in $16.80 \%$ of the included patients might be due to the coexistence of TXB2 and smoking history, and the risk of cerebral infarction caused by the coexistence of the two factors was 1.219 times as much as that caused by either of them alone and there was a positive synergy of the two factors; (2) the RERI of the interaction between the increased urinary TXB2 content and the hypertension history was $20.19 \%$, indicating that the risk of cerebral infarction caused by the increased TXB2 and the hypertension history was increased to 20.19 , the API was $78.87 \%$ and the S was 5.578 , indicating that $78.87 \%$ of cerebral infarction of the included patients was caused by the coexistence of increased TXB2 and hypertension history, and the occurrence of cerebral infarction caused by the coexistence of the two factors was 5.578 times as much as that of either of them alone and there was a positive synergy of the two factors; (3) the RERI of the interaction between the increased urinary TXB2 content and the diabetes history was 63.98 , indicating that the risk of cerebral infarction caused by the interaction between the increased TXB2 and the diabetes history was increased to $77.33 \%$, the API was 63.98 and the S was 4.604 , indicating that $77.33 \%$ of cerebral infarction of the patients was caused by the coexistence of increased TXB2 and diabetes history, and the risk of cerebral infarction caused by the coexistence of the two factors was 4.604 times as much as that caused by either of them alone and there was a positive synergy of the two factors. The incidence of cerebral infarction caused by the coexistence of increased 11-DH-TXB2 and the smoking history, hypertension history, or diabetes history was significantly higher than that caused by the smoking history, hypertension history or diabetes history alone, further demonstrating that there should be a statistically significant synergistic interaction between the urinary 11-DH-TXB2 and the other risk factors of cerebrovascular diseases. The change in the increased urinary 11-DH-TXB2 content in the early stage of cerebral infarction may be one of the risk factors for cerebral infarction. Reasonable and targeted application of drugs to reduce the content of 11-DH-TXA2 has a value in the prevention of cerebral infarction.

\section{ETHICS APPROVAL AND CONSENT TO PARTICIPATE}

The study was approved by Ethics Committee of Beihua University, China with approval number 25771589.

\section{HUMAN AND ANIMAL RIGHTS}

No animals were used in this research. All human research procedures followed were in accordance with the ethical standards of the committee responsible for human experimentation (institutional and national), and with the Helsinki Declaration of 1975, as revised in 2013. 


\section{CONSENT FOR PUBLICATION}

Written and informed consent was obtained from each participant prior to the study.

\section{AVAILABILITY OF DATA AND MATERIALS}

Not applicable.

\section{FUNDING}

This study was supported by Jilin Provincial Department of Health Research Project(2018J084), Jilin Science and Technology Bureau Medical and Health Key Projects (201830823), and Jilin Provincial Science and Technology Department (2016052014JH); Jilin Provincial Administration of Traditional Chinese Medicine (2018110), The $12^{\text {th }}$ Five-Year Plan for Scientific and Technological Research (Education Department of Jilin Province, JJKH 2014-508); Jilin City Science and technology innovation development Plan Project(201900982).

\section{CONFLICT OF INTEREST}

The authors declare no conflict of interest, financial or otherwise.

\section{ACKNOWLEDGEMENTS}

Declared none.

\section{REFERENCES}

[1] Wang L, Liu J, Ying Y, et al. Report on stroke prevention and treatment in China 2017 outline. Chin J Cerebrovasc Dis 2018; 15(11): 611-6.

[2] Chen J. Role of platelets in the formation and development of atherosclerosis. Chin Circ J 2004; 19: 5-6.

[3] Huang JS, Ramamurthy SK, Lin X, Le Breton GC. Cell signalling through thromboxane A2 receptors. Cell Signal 2004; 16(5): 521-33. [http://dx.doi.org/10.1016/j.cellsig.2003.10.008] [PMID: 14751539]

[4] Zhong W, Hu Y. Experimental study on atherosclerosisII: Effects of Chinese medicine 850 on the balance of thromboxane A2 and prostaglandin in rabbits with experimental atherosclerosis. Acta Academiae Medicinae Sinicae 1988; 10(6): 421-5. [PMID: 2978398]

[5] Borg C, Lim CT, Yeomans DC, et al. Purification of rat brain, rabbit aorta, and human platelet thromboxane A2/prostaglandin $\mathrm{H} 2$ receptors by immunoaffinity chromatography employing anti-peptide and antireceptor antibodies. J Biol Chem 1994; 269(8): 6109-16. [PMID: 8119956]
[6] Hui W, Dong W, Qi F, et al. Study on platelet activation in patients with hyperuricemia and hyperlipidemia. J Stroke Neurol Dis 2014; 31(7): 633-5.

[7] Wikström K, Kavanagh DJ, Reid HM, Kinsella BT. Differential regulation of RhoA-mediated signaling by the TPalpha and TPbeta isoforms of the human thromboxane A2 receptor: independent modulation of TPalpha signaling by prostacyclin and nitric oxide. Cell Signal 2008; 20(8): 1497-512.

[http://dx.doi.org/10.1016/j.cellsig.2008.04.006] [PMID: 18502100]

[8] Liu X, Fan Y, Gong Y. Reactivity of 11- dehydrothromboxane B2 to aspirin and its application in coronary heart disease. Chin J of Interv Cardiol 2018; 26(3): 167-71.

[9] Li L, Peng Y. Relationship between urinary 11-dehydrohematoxylin B2 and mortality in patients with stable coronary artery disease. Chin J Hypertens 2017; 25(1): 85.

[10] van der Plas A, Pouly S, de La Bourdonnaye G, Ng WT, Baker G, Lüdicke F. Influence of smoking and smoking cessation on levels of urinary 11-dehydro thromboxane $\mathrm{B}_{2}$. Toxicol Rep 2018; 5(5): 561-7. [http://dx.doi.org/10.1016/j.toxrep.2018.04.005] [PMID: 29854626]

[11] Li Z. JiSongbo. Relationship between the serum thromboxane B2 and 6-keto-prostacyclin F1 $\alpha$ levels and carotid atherosclerosis in patients with acute cerebral infarction. Chin J P Nerv Dis 2012; 15(4): 40-1.

[12] Chen J, Jiao L, Qin C, et al. Comparative Study on the antinociception of Zedoarydecoction, formulated granules and oil. Pharmacology and Clinics of Chinese Materia Medica 2016; 32(4): 60-3.

[13] Wang M, Lei Z, Zheng R, et al. Changes in the activity of thromboxane B2 and 6-keto-prostaglandin F1 $\alpha$, antithrombin III and plasminogen in experimental carotid atherosclerosis model. Chin J Atheroscler 2001; 9(2): 112-4.

[14] Qu Y, Jiang Z, Han T, et al. Correlation of platelet parameters and its activation markers with carotid intima-media thickness in type 2 diabetic patients. J Pers Med 2016; 32(8): 1250-3.

[15] $\mathrm{Hu}$ P. Clinical study on Bailing Capsule combined with cyclophosphamide and prednisone acetate in the Treatment of Nephrotic Syndrome. Drugs and Clinic 2016; 33(7): 1028-31.

[16] Zhu Y, Yi F, Liu G, et al. Analysis of the correlation between thromboxane A2 content and cerebral infarction. J Shanghai Jiaotong Univ 2009; 29(9): 1077-80.

[17] Xu En, Haixia Wen. Risk factors and intervention management of cerebrovascular diseases. Chin J Contemp Neurol Neurosurg 2015; 1 : 010.

[18] Katsnelson MJ, Della-Morte D, Sacco RL, et al. Stroke prevention: Recent achievements and new challenges. Period Biol 2012; 114(3): 409-19.

[19] Meschia JF, Bushnell C, Boden-Albala B, et al. American Heart Association Stroke Council; Council on Cardiovascular and Stroke Nursing; Council on Clinical Cardiology; Council on Functional Genomics and Translational Biology; Council on Hypertension. Guidelines for the primary prevention of stroke: A statement for healthcare professionals from the American Heart Association/American Stroke Association. Stroke 2014; 45(12): 3754-832.

[http://dx.doi.org/10.1161/STR.0000000000000046] [PMID: 25355 838]

(C) 2019 Lingling et al.

This is an open access article distributed under the terms of the Creative Commons Attribution 4.0 International Public License (CC-BY 4.0), a copy of which is available at: (https://creativecommons.org/licenses/by/4.0/legalcode). This license permits unrestricted use, distribution, and reproduction in any medium, provided the original author and source are credited. 